10 PRINZ, E.J., GARONE, P.M., SCHWARTZ, P.V., XIAO, X., and STURM, J.: 'The effect of base-emitter spacers and strain-dependent densities of states in $\mathrm{Si} / \mathrm{Si}_{1-x} \mathrm{Ge}_{x} / \mathrm{Si}$ heterojunction bipolar transistors'. Int. Electron Devices Meet. Tech. Dig., 1989, pp. 639-642

11 MANKU, T., and NATHAN, A: 'Effective mass for strained p-type $\mathrm{Si}_{1-}$ Ge', J. Appl. Phys., 1991, 69, (6), pp. 8414-8416

12 HONG, M., DE FRÉSART, E., STEELE, J., ZLOTNICKA, A., STEIN, C., TAM, G., RACANELLI, M., KNOCH, L., SEE, Y.-C., and EVANS, K.: 'Highperformance $\mathrm{SiGe}$ epitaxial base bipolar transistors produced by a reduced-pressure CVD reactor', IEEE Electron. Dev. Lett., 1993, 14, (9), pp. 450-452

13 NEUGROSCHEL, A., SAH, C.-T., FORD, J.M., STEELE, J., TANG, R., and STEIN, $\mathrm{C}$ : 'Comparison of time-to-failure of GeSi and Si bipolar transistors', IEEE Electron Dev. Lett., 1996, 17, (5), pp. 211-213

14 PEOPLE, R., and BEAN, J.C.: 'Band alignments of coherently strained $\mathrm{Ge}_{\mathrm{x}} \mathrm{Si}_{1-x} / \mathrm{Si}$ heterostructures on $<001>\mathrm{Ge}_{\mathrm{y}} \mathrm{Si}_{1-\mathrm{y}}$ substrates', Appl. Phys. Lett., 1986, 48, (2), pp. 538-540

15 GAN, C.H., DEL ALAMO, J.A., BENNETT, B.R., MEYERSON, B.S., CRABBÉ, E.F., SODINI, C.G., and REIF, R.: 'Si/Si ${ }_{-x} \mathrm{Ge}_{x}$ valence band discontinuity measurements using a semiconductor-insulatorsemiconductor (SIS) heterostructure', IEEE Trans. Electron Devices, $1994,41,(12)$, pp. 2430-2439

\section{Optimal design of P-buffer layer for GaAs power MESFET}

\author{
Y.J. Wang and C.C. Meng
}

\section{Indexing terms: MESFET, Power MESFET, Power transistors, Ion implantation}

The mechanisms for current conduction in the substrate of planar ion-implanted GaAs power MESFETs for wireless communication are investigated by $2 \mathrm{D}$ simulation. A properly doped $p$-buffer layer can eliminate the substrate conduction, but a too heavily doped $p$-buffer can reduce the saturation current $\left(I_{d s s}\right)$ and the breakdown voltage significantly. Optimal design for the $p$-buffer of the MESFETs is described.

Introduction: Ion-implanted planar GaAs power MESFETs have been widely used in commercial wireless communication [1, 2]. This structure intrinsically can be of low cost and manufactured in high volume. The power device is normally biased at low bias current for dual mode operation. The resulting power devices have high power-added-efficiency in the analogue mode, and have high linearity and good efficiency in the digital mode at the same bias voltage. However, the MESFET without the $p$-buffer has large leakage current in the substrate when the device is biased near pinch-off. This leakage current reduces the transconductance, drain efficiency and linearity. Hence, we need to know the physics of the leakage current by performing a $2 \mathrm{D}$ simulation.

In this study, two different mechanisms for leakage current in the substrate have been identified. One is called the "electron-pushout' effect and the other is the 'drain-induce-barrier-lowering (DIBL)' effect. The 'electron-push-out' effect happens just after the channel is pinched off. It acts as a resistor that is connected in parallel to the channel. The DIBL effect occurs as the gate is strongly reverse-biased. In the DIBL effect, the leakage current depends on the drain voltage exponentially. Both effects can be eliminated by a properly doped $p$-buffer in the substrate.

Simulation and results: $80 \mathrm{keV} 4.2 \times 10^{12} / \mathrm{cm}^{2} \mathrm{Si}$ implanted GaAs MESFETs with/without a $120 \mathrm{keV} 6 \times 10^{11} / \mathrm{cm}^{2}$ Be implanted $p$ buffer were analysed by $2 \mathrm{D}$ simulation. The devices had a gate length of $1 \mu \mathrm{m}$ and the gates had planar contours (i.e. there was no gate recess etching). Surface depletion of the devices was also accounted for in the simulation. The doping profile in the devices was as follows: A Gaussian distribution with $R_{p}=0.065 \mu \mathrm{m}$ and $\Delta R_{p}=0.037 \mu \mathrm{m}$ was used to simulate $80 \mathrm{keV}$ implanted $\mathrm{Si}(80 \%$ activation ratio); and a uniform distribution - 0.25 to $0.35 \mu \mathrm{m}$ from the surface with a $\Delta R_{p}=0.05 \mu \mathrm{m}$ Gaussian tail used to simulate the $120 \mathrm{keV}$ implanted $\mathrm{Be}(100 \%$ activation ratio). The parameters used here were based on the SIMS measurement.
The I-V curves simulated of an $80 \mathrm{keV}, 4.2 \times 10^{12} / \mathrm{cm}^{2} \mathrm{Si}$ implanted MESFET without the $p$-buffer are shown in Fig. 1. This device has a large leakage current near pinch-off as shown in region I of Fig. 1 and beyond pinch-off in region II. First, in region I, the mechanism of the leakage current can be clearly understood in Fig. 2, which shows the plot of the contours representing the distribution of different electron concentrations in the device for $V_{g s}=-3 \mathrm{~V}$ and $V_{d s}=4 \mathrm{~V}$ (just pinched-off condition). In Fig. 2, we can see that some electrons in the order of $10^{14}$ to $10^{15} /$ $\mathrm{cm}^{3}$ are pushed out from the depletion region under the gate and form an extra current path. We call it the 'electron-pushout' effect. This effect is not pronounced when the channel is opened but becomes significant when the channel is near pinch-off as can be seen in region I of Fig. 1. This undesired effect makes the substrate act as a parallel resistor and it smears out the knee voltage when the channel is near pinch-off.

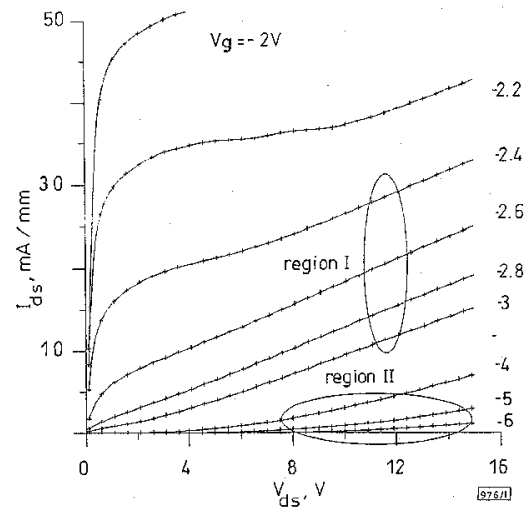

Fig. $1 \mathrm{I}$-V curves of $80 \mathrm{keV}, 4.2 \times 10^{12} / \mathrm{cm}^{2}$ Si implanted MESFET with out $p$-buffer

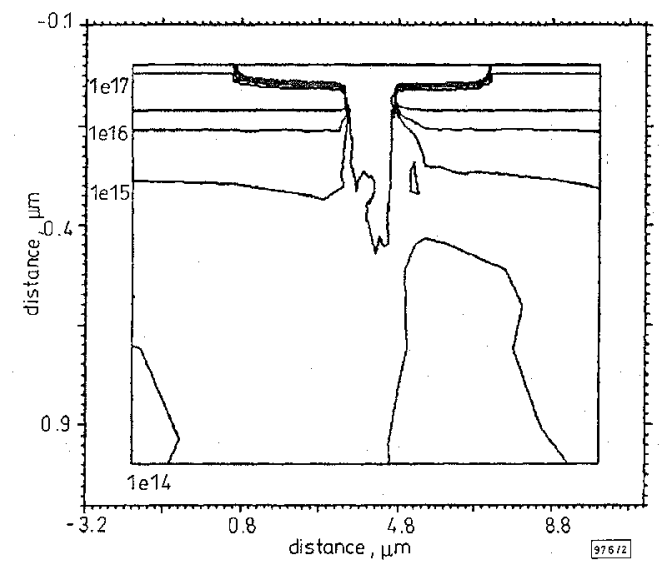

Fig. 2 Electron concentration contours of MESFET without p-buffer for $V_{g s}=-3 V$ and $V_{d s}=4 V$ (just pinched-off condition)

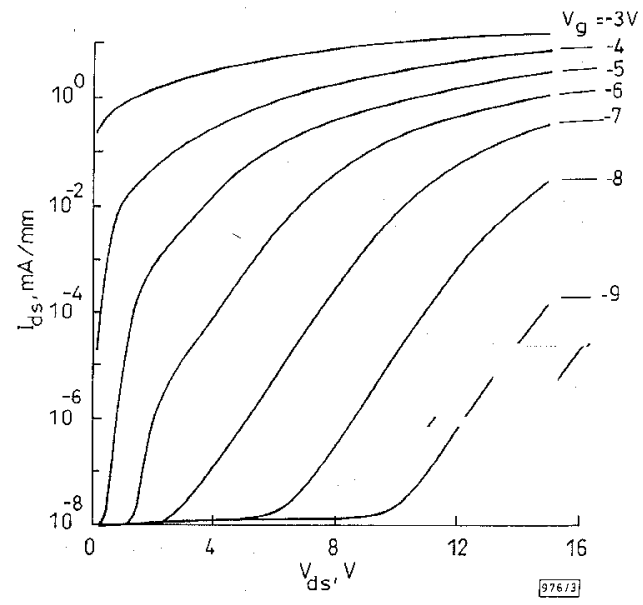

Fig. $3 \mathrm{I}-V$ curves of $80 \mathrm{keV}, 4.2 \times 10^{12} / \mathrm{cm}^{2}$ Si implanted MESFET without $p$-buffer

$I_{d s}$ in $\log$ scale 
Secondly, when the reverse gate bias is well beyond pinch-off, the device without the $p$-buffer still suffers from some small leakage current at large drain biases as shown in region II of Fig. 1. This can be interpreted by the DIBL effect as seen in Fig. 3. It shows the I-V curves of the same device as in Fig. 1, but its $y$-axis $\left(I_{d s}\right)$ is changed from a linear scale in Fig. 1, to a logarithmic scale. In Fig. 3, the DIBL effect is obvious, because the drain leakage current increases exponentially as the drain voltage increases. This exponential dependence is evidence of the DIBL effect. Since the gate potential barrier is lowered by the drain voltage, the leakage current increases exponentially. The DIBL effect can be also verified in the 2D simulation by observing the difference of the potential contours between different drain biases beyond pinch-off. Detailed descriptions for these are not included in this Letter.

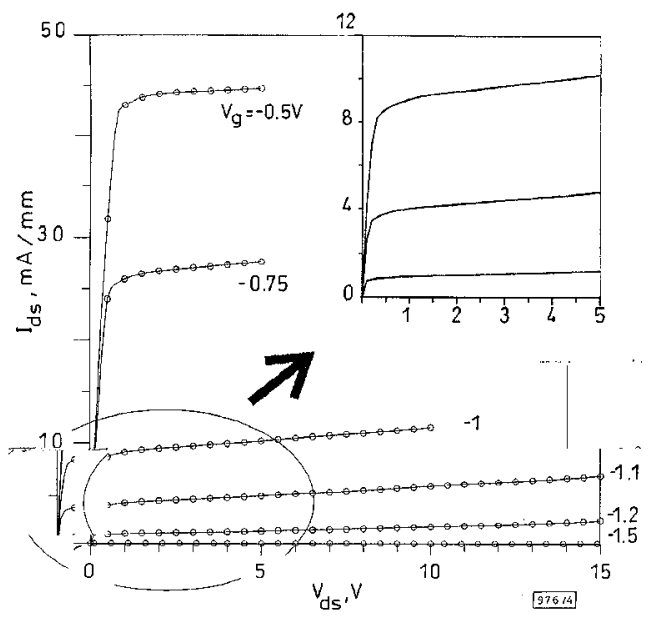

Fig. $4 \mathrm{I}$-V curves of $80 \mathrm{keV}, 4.2 \times 10^{12} / \mathrm{cm}^{2}$ Si implanted MESFET with $120 \mathrm{keV}, 6 \times 10^{11} / \mathrm{cm}^{2}$ Be implanted p-buffer

As for the MESFET with the $p$-buffer, the 'electron pushed out' and the DIBL effect both disappear. Fig. 4 shows the I-V curves

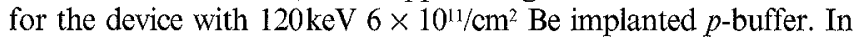
Fig. 4, we can see that there is no leakage current near pinch-off and the I-V characteristics have well-defined knee voltages even near pinch-off. Moreover, the electron concentration contours of the device are also plotted. It can be seen that the electrons were confined in the channel very well by the $p$-buffer, both for the before-pinch-off condition and the beyond-pinch-off condition. The $p$-buffer can prevent electrons from being pushed out into the substrate when the gate voltage is before or near pinch-off; and can strengthen the potential barrier and consequently reduce the DIBL effect when the gate voltage is beyond pinch-off.

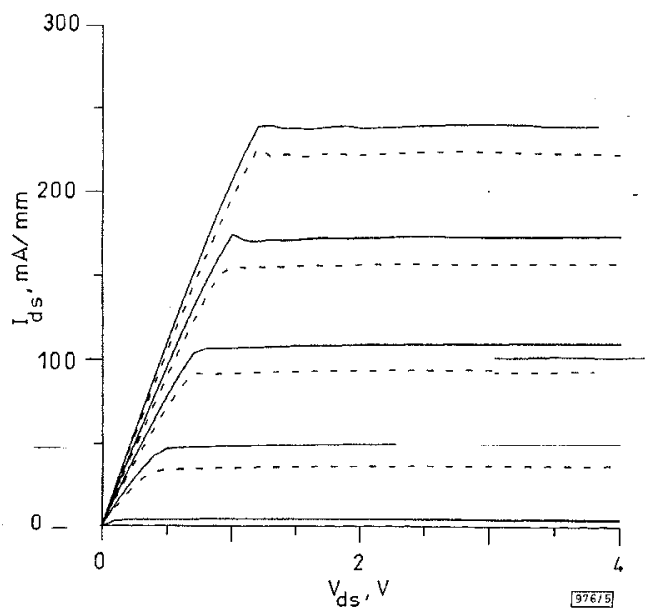

Fig. $5 \mathrm{I}-\mathrm{V}$ curves of $100 \mathrm{keV}, 6 \times 10^{12} / \mathrm{cm}^{2}$ Si implanted MESFET

MESFET with $1.5 \times 10^{12} / \mathrm{cm}^{2}$ Be implanted $p$-buffer - - - MESFET with $2.2 \times 10^{12} / \mathrm{cm}^{2}$ Be implanted $p$-buffer top line: $V_{g}=0$

$-0.5 \mathrm{~V} / \mathrm{step}^{3}$

There are some disadvantages of the $p$-buffer. Although the $p$ buffer can eliminate the substrate leakage, the $I_{d s s}$ of the MESFET is also reduced by the $p$-buffer. For example, the $I_{d s s}$ of the two devices discussed above is reduced from 230 (without $p$-buffer) to $100 \mathrm{~mA} / \mathrm{min}$ (with $p$-buffer). To obtain the desired $I_{d s s}(\sim 200$ $250 \mathrm{~mA} / \mathrm{mm}$ ), we should try a heavier dosage of the Si $n$-type dopant. In addition, the energy of the implanted $\mathrm{Si}$ ion must also be increased in order to obtain nearly the same peak concentration of doping. Therefore, $100 \mathrm{keV} 6 \times 10^{12} / \mathrm{cm}^{2} \mathrm{Si}$ implanted MESFETs with a $120 \mathrm{keV}$ Be implanted $p$-buffer were used. Two Be dosages were chosen, $a 1.5 \times 10^{12} / \mathrm{cm}^{2}$ and $b 2.2 \times 10^{12} / \mathrm{cm}^{2}$. The $\mathrm{I}-\mathrm{V}$ curves of the two MESFETs are shown in Fig. 5. The $I_{d s s} s$ of the two devices are $a 240 \mathrm{~mA} / \mathrm{mm}$ and $b 220 \mathrm{~mA} / \mathrm{mm}$. Both devices show very little leakage current.

The $p$-buffer also affects the breakdown phenomena. If the $p$ buffer was not totally depleted, we found by simulation that the avalanche breakdown occurred easily at the junction of the $n$ channel and the $p$-buffer. Hence, it was called 'substrate breakdown'. Whenever the substrate breakdown occurred, as a result, the breakdown voltage was greatly reduced. For example, in device $a$, in which we found by simulation that the $p$-buffer was totally depleted under drain, no substrate breakdown occurred. The breakdown phenomenon was just like the phenomena in devices without $p$-buffer, which occur at the gate edge close to the drain. Conversely, in device $b$, in which the $p$-buffer was not totally depleted under the drain, avalanche breakdown occurred at the $p n$ junction under the drain. Thus, substrate breakdown occurred. Consequently, the breakdown voltage was greatly reduced. Therefore, the breakdown phenomenon puts an upper limit on the Be dosage of the $p$-buffer.

Conclusion: Determined by 2D simulation, the optimal $p$-buffer for a GaAs MESFET can be obtained by carrying out $120 \mathrm{keV} 6 \times$ $10^{11 / \mathrm{cm}^{2}}$ silicon ion implantation with $100 \mathrm{keV} 1.5 \times 10^{11} / \mathrm{cm}^{2}$ beryllium ion implantation. The resulting device has no substrate breakdown because the $p$-buffer is totally depleted. In addition, the device does not have substrate leakage. In conclusion, an ionimplanted planar power MESFET with adequate $I_{d s s}$, high breakdown voltage and little substrate leakage current, has been successfully achieved by carrying out a $2 \mathrm{D}$ simulation.

(C) IEE 1996

7 October 1996

Electronics Letters Online No: 19961509

Y.J. Wang and C.C. Meng (Room 451, Electric Engineering Department, National Taiwan University, Taipei, Taiwan, Republic of China)

\section{References}

1 MILLER, D.C., SADLER, R.A., and PEAKER, A.H.: 'Robust GaAs MMIC amplifier using planar ion-implanted power MESFETs with improved open-channel burn-out'. IEEE MTT-S Symp., 1995,

2 Masato, H., MaEda, M., and FuJimoto, H.: 'Analogue/digital dual power module using ion-implanted GaAs MESFETs'. IEEE MTTS Symp., 1995

\section{Quasi-Schottky contacts on $n-\ln _{0.35} \mathrm{Ga}_{0.65} \mathrm{As}$ epitaxial layers deposited on $\mathrm{GaAs}$ substrates}

E. Gombia, R. Mosca, A. Motta, H. Chaabane,

A. Bosacchi and S. Franchi

Indexing terms: Schottky barriers, Indium compounds, Gallium arsenide

Al Schottky barriers have been prepared on $n-\mathrm{In}_{0.35} \mathrm{Ga}_{0.65} \mathrm{As}$ / buffer/GaAs structures using $\operatorname{In}_{x} \mathrm{Ga}_{1-x}$ As buffers with different grading laws to reduce the defect density induced by lattice mismatch in the active $\operatorname{In}_{0.35} \mathrm{Ga}_{0.65}$ As layer. Counter doped $p^{+}$cap layers have been used to enhance the barriers. Using suitable cap and buffer parameters, barrier heights up to $0.67 \mathrm{eV}$ and ideality factors of 1.15 have been obtained. 